\title{
Flessibilità e adattamento al cambiamento nella trasposizione didattica a distanza
}

\author{
Flexibility and adaptation to change to improve teaching trans= \\ position in distance education \\ Michela Galdieri", Michele Domenico Todino**, Alessia Scarinci***
}

\begin{abstract}
Riassunto
Il lavoro presenta una lettura in chiave semplessa del processo di insegnamentoapprendimento nell'ambito della didattica a distanza e una riflessione sulle dinamiche educative ridefinite dalle tecnologie digitali. I mutati scenari sociali, politici ed economici correlati al sopraggiungere dell'inaspettata emergenza sanitaria, hanno sollecitato, anche in ambito educativo, la ricerca di pratiche didattiche flessibili ed innovative, la rivisitazione dei tempi e dei luoghi della didattica e la rimodulazione del modus operandi del docente la cui professionalità si esplica nel suo ruolo proattivo, trasformativo e nella sua capacità, talvolta, inconsapevole, di destrutturare itinerari conoscitivi ed esperienziali generando nuove ed originali forme di agire didattico. L'esperienza svolta presso l'Università degli Studi del Sannio di Benevento nel percorso formativo dei $24 \mathrm{CFU}$ attraverso la somministrazione di un questionario a centottantatré studenti, ed estesa anche a cinquantadue docenti campani, disciplinaristi e non di sostegno, propone una riflessione sul tema della trasposizione didattica in modalità e-learning a partire da una progettualità educativo-didattica in linea con il bisogno del discente di autodeterminazione dei propri apprendimenti.
\end{abstract}

Parole chiave: professionalità docente, agire didattico, e-learning, tecnologie, semplessità, trasposizione didattica.

\section{Abstract}

In this paper will be described how simplexity could improve distance education teaching-learning process through different educational behaviors and

\footnotetext{
${ }^{*}$ Docente a contratto di Didattica e pedagogia dell'inclusione presso l'Università degli Studi del Sannio di Benevento. E-mail: michela.galdieri1@istruzione.it.

** Docente a contratto di Tecnologie didattiche per l'inclusione presso l'Università degli Studi del Sannio di Benevento. E-mail: micheledomenico.todino@istruzione.it

${ }^{* * *}$ Ricercatrice presso 1' Università degli Studi di Bari Aldo Moro. E-mail: alessia.scarinci@uniba.it.

I tre autori hanno condiviso il tema dell'articolo. Tuttavia, si specifica che Michela Galdieri ha redatto i paragrafi 1, 2, 6, Michele Domenico Todino ha redatto i paragrafi 3,5 e Alessia Scarinci il paragrafo 4 .
} 
approaches using digital technologies. These new social, political and economic scenarios due to this unexpected health emergency, ask teachers to examine how to be flexible in several ways, such as: using innovative teaching practices to review teaching times and places, changing modulation of daily working habits, rescheduling and planning activities, improving a proactive and transformative professional working role due to an unconscious ability to deconstruct cognitive and experiential itineraries and, last but not least, generating new and original practices of didactical actions. To support this research, there was gathered data from an experience carried out at the University of Sannio during the training course of $24 \mathrm{CFU}$, using a survey that concerned didactic transposition in elearning modality preparatory from an educational-didactic planning to prepare course final exam; these survey was compiled to one hundred eighty-three students and also submitted from other fiftytwo teachers of Campania region to whom data collection purpose was explained.

Keywords: teaching profession, teaching agency, e-learning, technology, simplexity, didactic transposition.

Può, il batter d'ali di una farfalla in Brasile, provocare un tornado in Texas? Edward Lorenz, 1972

\section{Una visione d'insieme}

È ormai idea condivisa che la comprensione della realtà fenomenica, nelle sue aggrovigliate trame, possa avvenire attraverso approcci volti ad analizzare non unità isolate, ma la realtà generata dall'interazione di tutte le componenti di un sistema. Il paradigma scientifico classico tentava di isolare gli elementi dell'universo osservato sperando «che si potesse ottenere, rendendolo intellegibile, il complesso, ovvero il sistema, e poteva trattarsi della cellula, della mente o della società» (von Bertalanffy, 1983, p.13). Sin dalla sua formulazione originaria il concetto di sistema, invece, è utilizzato per indicare «un complesso di componenti in interazione, un tutto formato da parti collegate fra loro in un certo modo, un insieme di unità in relazione» (Miller, 1986, p.16). I nuovi assetti epistemici vertono su ciò che è definito, con una certa imprecisione, "totalità", su problemi di organizzazione, su fenomeni apparentemente non più risolvibili in eventi locali, su interazioni dinamiche che appaiono nella differenza 
di comportamento delle parti, su sistemi di diversi ordini che non sono comprensibili mediante studi delle loro componenti singole ed isolate.

Queste suggestioni che permeano una pluralità di settori scientifici, a partire dalla prima metà del secolo scorso, sollecitano una visione olistica della conoscenza, allontamento da schemi mentali irrigiditi e infecondi e «lasciano il podio a percorsi illineari, marcati dal problematico e dalla continua ricombinazione degli elementi conoscitivi frammentari ed occasionali. Esplode la dimensione del "possibile", che coinvolge il sapere, il pensiero e le scienze contemporanee. Il sapere si ispira al possibile o potenziale, anzichè al lineare e al prevedibile» (Giaconi, 2008, p. 51) e la prospettiva che inizia a delinearsi «è quella di un rapporto costruttivo, circolare e vicariante fra vincolo e possibilità (...). Non soltanto la natura ha una storia: sono il possibile e il necessario, anche, ad avere una storia» (Ceruti, 2000, p.19).

I confini tra le scienze della natura e le scienze umane appaiono sempre più labili e i sistemi sono indagati nella complessità del loro essere e delle loro relazioni: «Tutte le varie complessità si intrecciano, dunque, e si tessono insieme, per formare l'unità della complessità; ma l'unità del complexus non viene con ciò eliminata dalla varietà e dalla diversità delle complessità che l'hanno tessuto» (Morin, 1995, p.56). Il pensiero complesso riconosce «un'inadeguatezza sempre più ampia, profonda e grave tra i nostri saperi disgiunti, frazionari, suddivisi in discipline da una parte, e realtà o problemi sempre più polidisciplinari, trasversali, multidimensionali, transnazionali, globali, planetari dall'altra» (Morin, 2000, p.5), e per queste ragioni, invita ad un'interdisciplinareità dei saperi senza tuttavia tralasciare lo studio dei dettagli disciplinari che sembra essere, al contrario, uno dei modi possibili per fare emergere nuove proprietà e significatività. Allo stesso modo «per la didattica, e le discipline pedagogiche, vale ovviamente quanto detto per le scienze umane in generale, ovvero la promessa di disporre di una epistemologia unificata, comune e articolata per un dominio della ricerca scientifica» (Damiano, 2008, p. 17). La nuova visione circolare e dinamica della conoscenza, che riconosce le interazioni alla base di meccanismi fisici, biologici, psicologici o sociali, non solo arricchisce di nuovi e più ampi significati $i$ contenuti delle scienze tradizionali ma prospetta, anche in ambito educativo e per la didattica, itinerari di ricerca e di rifessione orientati alla convergenza e alla dialogicità tra i saperi:

I temi classici della ricerca pedagogica richiedono specifiche curvature, rese necessarie dalla complessità della situazione socio-culturale e dai contributi di nuove discipline. Si modifica anche il ruolo del docente, che oggi, più che in passato, deve porsi nell'ottica di un professionista dell'educazione, capace di operare in contesto, proponendo per le situazioni problematiche soluzioni legate al qui e ora (Rossi, 2011, p.11). 
L'emergenza epidemiologica, ancora in atto, ha senza ombra di dubbio generato un disequilibrio negli scenari politici ed economici internazionali, cambiamenti significativi nelle dinamiche sociali e relazionali, affermando la sua natura di "situazione problematica" di portata planetaria nella misura in cui ha oltremodo rinvigorito aspetti della complessità del reale, una complessità che «si presenta con i lineamenti inquietanti dell'accozzaglia, dell'inestricabile, del disordine, dell'ambiguità, dell'incertezza» (Morin, 1993, p.10): il mondo appare irriconoscibile, le sue logiche incomprensibili, i destini comuni ribaltati da un battito d'ali a riprova di come un singolo, seppur lontano evento, possa produrre effetti che si traducono in comportamenti ed azioni non sempre prevedibili con esattezza (Bocchi e Ceruti, 1995; Morin, 1995).

In questi mutati scenari, le dinamiche di insegnamento-apprendimento, corroborate, già di per sè, da un numero talvolta incalcolabile di variabili legate al contesto, alla pluralità di stili cognitivi e processi apprenditivi dei discenti, alla presenza di bisogni emergenti nelle eterogeneità delle classi hanno dovuto ancora una volta accogliere e fronteggiare perturbazioni esogene alla relazione educativa che, come incontrollabili forze propulsive, hanno richiesto nuove ed inaspettate ristrutturazioni dei tempi, dei modi e dei luoghi della didattica. Ma nello spazio del caos e nel tempo della complessità (Ceruti, 2018) «quale conoscenza, quali competenze, quali apprendimenti, quali professionalità sono necessarie per far fronte alle sfide che il nuovo secolo presenta?» (Fabbri, 2014, p.351). È possibile adattarsi al cambiamento, rinnovarsi senza snaturasi? «Semplicemente agendo, giocando d'anticipo» (Aiello P., Sharma U. e Sibilio M., 2016, p.15).

È sulla base di queste premesse che si propone una riflessione, in chiave semplessa, sul ruolo proattivo e trasformativo del docente, sulla sua capacità di destrutturare itinerari conoscitivi ed esperienziali generando nuove ed originali forme di azione didattica dimostrando «flessibilità e adattabilità al cambiamento» (Berthoz, 2011, p. 9). Si rintracciano, inoltre, le origini del suo intenzionale agire nelle più recenti tradizioni di ricerca, consapevoli che la relazione apprendimento-formazione «non può essere compresa se non attraverso dimensioni pluridisciplinari che facciano delle recenti acquisizioni neuroscientifiche una fondamentale chiave interpretativa e le includano, attraverso le scienze bieducative, nel più ampio discorso delle scienze dell'educazione» (Orefice e Sarracino, 2006, p.38; Frauenfelder,1983, 1994), un discorso in cui anche la didattica, come scienza dell'insegnamento, è chiamata «ad occuparsi centralmente, non in forma residuale o di "nicchia", dei fenomeni complessi della mente (...) una teoria didattica epistemologicamente estranea alle ricerche e alle teorizzazioni sui processi mentali ed evolutivi in genere, non può che configurarsi come una "didattica cieca", che si priva dell'utile confronto con il "cielo stellato"»" 
(Crispiani, 2008, p.28). La seconda parte del contributo, è incentrata sull'esperienza svolta presso l'Università degli Studi del Sannio nel percorso formativo dei $24 \mathrm{CFU}$, e propone un esempio di trasposizione didattica in modalità elearning in cui si declinano, principi regolatori della teoria semplessa a partire da una progettualità educativo-didattica in linea con il bisogno del discente di autodeterminazione dei propri apprendimenti.

\section{Complessità e semplessità dell'agire didattico}

L'epistemologia contemporanea, nel proporre modelli non lineari della ricerca scientifica, ha spostato il focus su una visione della conoscenza maggiormente rispondente alla complessità dei suoi oggetti di studio intellegibili a partire dall'analisi delle dinamiche interattive e circolari che non solo li definiscono internamente, ma ne sostanziano anche il rapporto con l'esterno. I sistemi viventi non compiono mere azioni adattive ma instaurano ricorrenti interazioni con l'ambiente che «costituiscono perturbazioni reciproche. In questa interazione la struttura dell'ambiente innesca solamente i cambiamenti strutturali delle unità autopoietiche (non li determina nè li 'istruisce') e lo stesso avviene per l'ambiente» (Maturana e Varela, 1999, p.80). L'accoppiamento strutturale che ne deriva non è solo l'esito di una storia di reciproci mutamenti ma anche di impliciti atti cognitivi: è il soggetto agente che, a partire dalla sua determinazione interna, opera una selezione e procede verso un'attribuzione di senso agli stimoli e alle direzioni dei cambiamenti strutturali in funzione di una conservazione della propria identità «è l'attore a specificare in via prioritaria cosa dell'ambiente lo perturba e cioè ne tiene conto nella sua attività sulla base dei suoi stati di accoppiamento successivi nel passato (...). L'attività di un attore è dunque situata, o meglio 'fenomenologicamente situata', nel senso che essa esprime il vissuto e il punto di vista di quest'attore nel suo ambiente, ovvero sulla sua 'situazione'» (Durand e Poizat, 2017, pp. 31-33).

L'approccio enattivo nel riconoscere che ogni conoscenza è accompagnata sempre da schemi di azioni e «il vivere in quanto processo è un processo di cognizione» (Maturana e Varela, 1985, p.59), ci restituisce una visione dell'esperienza conoscitiva radicata nella struttura biologica dell'individuo e sollecita la didattica ad indagare le potenzialità del corpo in azione "per produrre modalità di significazione alternative, complementari e vicarianti» (Sibilio, 2017, p. 54), in linea con i contributi delle nuove scienze cognitive (Lakoff e Johnson, 1999; Johnson, 2017; Borghi e Iachini, 2004) secondo cui «tra processi percettivi, cognitivi e motori non vi è un rapporto gerarchico o di sequenzialità temporale (prima percepiamo un evento, poi pensiamo a come agire, e alla fine lo facciamo) ma piuttosto una circolarità tale per cui l'azione influenza 
sia la percezione che il pensiero astratto» (Caruana e Borghi, 2016, p. 20). Mentre la percezione implica la costruzione di una rappresentazione del mondo esterno, «l'azione, invece, comincia con un'ipotesi sulle conseguenze desiderate di un movimento (...). Agire, vale a dire compiere movimenti, significa quindi stabilire inizialmente una mappa della realtà attraverso coordinate che dipendono da una serie di parametri sensoriali e spaziali» (Oliverio, 2017, p. 22). Le evidenze scientifiche dimostrano come l'esperienza della conoscenza, a partire dal coinvolgimento di diverse strutture cerebrali e corporee, coinvolga il soggetto percepiente in maniera attiva e in un modo del tutto personale: l'azione, che assume un ruolo centrale negli studi sulla didattica, nella prospettiva di Damiano, si identifica infatti come

un processo che si svolge nel tempo, in grado, più o meno intenzionalmente, di indurre dei cambiamenti (...), un'azione è un pensiero incarnato, essa ha un legame immanente con le intenzioni di chi la compie, con i suoi intenti, la sua sensibilità, la sua condizione. Per conoscere un'azione, veramente, non ci si può limitare ad osservarla in superficie, per quel che si vede, ma comporta rendere conto della parte di soggettività iscritta nella sua fisicità, cultura, trama di relazioni significative (Damiano, 2008, pp. $17-18)$.

L'agire è, dunque, intriso di soggettività, ogni azione ed ogni esperienza assumono caratteristiche di unicità e di irripetibilità, sono la risposta sensomotoria dell'individuo e il risultato tangibile di una pianificazione mentale per il raggiungimento di uno scopo oltre che di un evidente atto interpretativo del reale: nella sua funzione di "decodificatore del mondo" il cervello interpreta il nostro comportamento e le nostre risposte agli stimoli ambientali e «stabilisce costantemente un processo narrativo delle nostre azioni, emozioni, sogni, pensieri. È il collante che unifica la nostra storia e crea la nostra percezione di essere un agente razionale completo» (Gazzaniga, 2007 p.38); nel suo essere un anticipatore, opera in modo automatico e principalmente al di fuori della nostra consapevolezza «generando in noi l'illusione che gli eventi che stiamo sperimentando accadano in tempo reale e non prima che noi decidiamo coscientemente di fare qualcosa» (Gazzaniga, 1999, p. 79); nel suo essere un simulatore, ipotizza, crea modelli e soluzioni che proietta sul mondo: «il cervello interroga i recettori regolando la sensibilità, combinando i messaggi, prespecificando i valori stimati, in funzione di una simulazione interna delle conseguenze attese dall'azione» (Berthoz, 1998, p. 253). Queste potenzialità cognitive implicite consentono al docente di orientare il proprio agire in funzione di scopi ed obiettivi, di pianificare i propri atti traspositivi maturando la consapevolezza che 
Senza dubbio ogni qual volta che il nostro cervello anticipa un'azione compare uno stato differente. E come la percezione è sempre simulazione di un'azione nel mondo, la percezione cosciente è sempre un'anticipazione di un qualche evento che si produrrà nel mondo, a prescindere dal fatto che l'evento sia prodotto dal soggetto percepente oppure no. La coscienza non è la coscienza di quello che facciamo, visto che abbiamo coscienza dopo aver anticipato. Io dico che siamo coscienti di qualcosa che anticipiamo (Berthoz, 2011, p. 173).

L'agire didattico diventa, dunque, riflesso di funzioni decodificatrici e interpretative, la risultante di selezioni e scelte di informazioni, sintesi di processi attentivi e mnestici, esito di schemi di anticipazione visuomotoria e di strategie decisionali e la didattica una neurodidattica il cui scopo non è quello di «indicare agli insegnanti la correlazione tra funzionamento del cervello e automatica soluzione dei problemi didattici in classe, ma individuare proprio attraverso lo studio del cervello spazi proficui per la ricerca didattica» (Rivoltella, 2012, p. IX). Gli itinerari di ricerca in ambito educativo nell'accogliere queste suggestioni, indagano i meccanismi attenzionali, percettivi e decisionali della persona, indispensabili nella soluzione di situazioni problematiche, talvolta inaspettate, e riconoscono le capacità semplesse del docente, laddove per semplessità si intende «questo insieme di soluzioni trovate dagli organismi viventi affinché, nonostante la complessità dei processi naturali, il cervello possa preparare l'atto e anticiparne le conseguenze» (Berthoz, 2011, p. XI).

Nei principi della fisiologia della percezione e dell'azione è possibile rintracciare il significato delle capacità predittive richieste ai docenti dal momento che «sulla previsione si costruisce anche la capacità del formatore di "tenere" l'aula: nell'ottica di una vera e propria semiotica dello spazio, saper leggere i sintomi nei volti, negli sguardi, nelle dinamiche, significa immaginare cosa potrebbe succedere e agire di conseguenza» (Rivoltella, 2014, pp. 5-6). Il pensiero semplesso che non semplifica nè riduce, ma induce a selezionare, scegliere, collegare e immaginare mondi non ancora creati offre alla didattica opportunità per ripensarsi in maniera originale e creativa, per sollecitare il docente ad assumere un atteggiamento flessibile e adattabile al cambiamento anche in quelle circostanze in cui gli eventi esterni, improvvisi ed inaspettati richiedono una rivisitazione delle partiche di insegnamento ed esigono una conversione della trasposizione dell'atto educativo da "atto in presenza" ad "atto a distanza".

\section{Dalle lezioni in presenza alla didattica a distanza}

Prima dell'emergenza sanitaria si avvertivano, da parte dei docenti, alcune resistenze nei confronti della didattica a distanza, emerse da numerosi studi di Media Education (Rivoltella, 2012, 2013, 2014, 2015, 2020), e generalmente 
la didattica si svolgeva quasi unicamente in presenza ovvero nelle aule, nei laboratori, ma anche nei corridoi alternando aspetti formali, non formali e informali (Pellerey, 2002, 2011; Sibilio, 2020) del processo di insegnamento-apprendimento. Questa situazione aveva origine principalmente dal fatto che da sempre lo spazio occupa un ruolo centrale nella storia dell'educazione (Sibilio e Zollo, 2016) che avviene, innanzitutto, nelle classi e nelle scuole che assieme alla strutturazione temporale hanno fortemente influenzato l'organizzazione della didattica, di conseguenza ogni ambiente per essere realmente educativo deve essere organizzato spazialmente (e temporalmente), analizzando le dinamiche prossemiche che regolano il processo di insegnamento-apprendimento e la comunicazione educativo-didattica (Ibidem). Durante, e probabilmente dopo, l'emergenza sanitaria, opposizioni, resistenze e rifiuti che per anni erano emerse nei riguardi della didattica a distanza, delle opportunità offerte da didattiche blended e flipped, saranno almeno in parte superate dai docenti, di ogni ordine e grado, e dagli studenti. Questa esperienza collettiva ha permesso a tutti di vivere una situazione singolare, che ha visto mutare gli spazi entro i quali svolgere quotidianamente le proprie attività. Volendo descrivere meglio questa nuova quotidianità, da marzo 2020 i tempi della didattica sono stati scanditi dai calendar (di Microsoft Teams, GSuite for Education, Zoom, etc.) e i luoghi sono divenuti piattaforme digitali (Edmodo, WeSchool, Moodle, etc.), spazi virtuali, sistemi multimediali e soluzioni multimodali. L'impossibilità di accedere negli edifici scolastici ha posto il problema di come ricreare situazioni laboratoriali; per fronteggiare questa situazione molti docenti si sono affidati a software didattico-disciplinari che prima non utilizzavano, perché avevano l'attrezzatura fisica necessaria giustappunto nei propri laboratori. Solo per menzionare due casi, riferiti alle scuole secondarie di secondo grado, si possono rammentare: Cisco Packet Tracer che ha preso il posto del laboratorio di informatica nei licei tecnologici e negli istituti tecnici industriali e Human Anatomy Atlas 2021: Complete 3D Human Body che ha permesso di continuare le attività laboratoriali di scienze integrate nei licei scientifici e tecnologici. Oltre a queste soluzioni tecniche, il periodo di didattica a distanza forzata, se "da un lato" ha diviso fisicamente insegnanti e studenti "dall'altro" ha rimodulato le loro relazioni didattiche.

In particolare, il cambiamento, dovuto alla didattica a distanza, ha richiesto al docente di risintonizzare la trasposizione didattica. È importante ricordare che la trasposizione didattica si divide in esterna e interna: la prima «è quella realizzata dalle commissioni ideatrici dei programmi ministeriali e da coloro che redigono i manuali scolastici» (Rossi e Pezzimenti, 2014, p.170); la seconda, definita trasposizione didattica interna, di cui si occuperà questo articolo, «è quella compiuta dagli insegnanti» (Ibidem) per realizzare la corrispondenza tra il proprio agire didattico e la declinazione armonica ed efficace delle 
proprie risorse in relazione ai bisogni del processo di insegnamento-apprendimento (Sibilio e Zollo, 2016), per permettere ai savoir savant di divenire saperi insegnati, come descritto nel dettaglio da Chevallard (Nigris, 2014; Rossi e Pezzimenti, 2014). Tale processo avviene attraverso «un'azione di selezione, di scelta dei contenuti che il docente (...) ritiene irrinunciabili dal punto di vista epistemologico e più idonei dal punto di vista del senso che possono acquisire rispetto al mondo» (Rossi e Pezzimenti, 2014, p. 170). Più nel dettaglio, la trasposizione didattica è fondamentale nel processo di insegnamento-apprendimento e potrebbe essere descritta secondo varie prospettive che principalmente sono quattro, ci sono autori che:

1) «pongono a fondamento del processo di trasposizione l'epistemologia del sapere e la psicologia dell' apprendimento» (Ivi, p. 167) in questi casi il docente, nella sua didattica a distanza dovrà tenere sotto controllo questi elementi;

2) «mettono l'accento sulla filosofia educativa del docente» (Ibidem) che guiderà le lezioni;

3) «leggono la trasposizione come mediazione» (Ibidem) facendo perno sugli elementi relazionali;

4) «focalizzano l'attenzione anche sulla situazione didattica» (Ibidem). In tutti questi casi, la didattica a distanza può essere un limite ma può anche offrire nuove potenzialità.

La didattica a distanza, oltre alla trasposizione didattica ha "colpito al cuore" i tradizionali spazi dell 'interazione didattica che erano la sintesi tra prossemica e comunicazione (Sibilio e Zollo, 2016; Sibilio, 2020). Tali spazi dell'interazione, eredi di lunga tradizione educativa che fondava le sue radici nella paideia, tenevano conto della distanza interpersonale che a sua volta prendeva spunto dagli elementi che guidano le relazioni private, sociali e istituzionali (Hall, 1969; Argyle, 1992) applicate di conseguenza nelle scuole e nelle università. Come sono cambiati tali spazi con la didattica a distanza? Di seguito un parziale sommario:

1) le distanze dagli strumenti impiegati nella trasposizione didattica: lavagne, schermi e supporti tecnologici (Sibilio e Zollo, 2016), si comprimono su un unico schermo che è ora nelle mani del discente e determinate dal docente che proietta la sua lezione condividendo il suo schermo;

2) la grandezza dei caratteri e delle icone (Ibidem) si impostano direttamente dall'utente producendo numerosi benefici in termini di accessibilità digitale;

3) le posizioni degli studenti in relazione al ritmo e agli spostamenti del docente (Ibidem) si riducono a piccoli rettangoli tutti uguali organizzati a griglia sul monitor dell'insegnante;

4) «la forma statica e dinamica del corpo del docente» (Ibidem) si limita drasticamente riducono l'importanza del corpo in movimento nell'agire-didattico (Sibilio 2011, 2012a, 2012b, 2014a, 2014b, 2015). 
Per ovviare a questi ultimi due casi, l'applicazione per smartphone Kinoni permette di integrare varie webcam ad un'unica videoconferenza aumentando le inquadrature, sia del docente sia degli studenti, permettendo l'uso di prospettive multiple della stessa persona e dello spazio nel quale la persona è immersa realmente. In tal modo il docente non è più costretto a sedersi davanti al suo device; nel caso specifico delle discipline motorie e sportive, esse possono avvalersi di questa applicazione incrementando notevolmente il successo formativo anche attraverso la didattica a distanza. L'applicazione Kinoni permette di progettare soluzioni spaziali alternative a quella in cui docente e discenti sono tutti seduti davanti a un computer, in questo modo è possibile un differente uso della comunicazione verbale, in relazione al ritmo ed agli spostamenti del docente e il corpo può essere considerato come uno spazio simbolico capace di rappresentare una forma alternativa di significazione (Sibilio e Zollo, 2016); è importante che il corpo del docente e quello dei discenti non siano ridotti, per diminutio, a un viso compresso su di uno schermo bidimensionale rinchiuso in un rettangolino, rievocando, per suggestione Flatlandia il Racconto fantastico a più dimensioni di Abbott. Oggigiorno, non solo gli studenti ma anche i docenti sono chiamati, a fortiori, «ad attraversare infiniti mondi. Particolarmente al presente, e maggiormente nel futuro. Caratterizzate come sono, queste due dimensioni temporali, da trasformazioni continue e spesso impreviste e imprevedibili, che corrono con la velocità della luce. Al pari della loro attuale, e futuribile, articolazione complessa» (Corsi, Stramaglia e Gellel, 2019, p.8).

\section{La flessibilità e l'adattamento al cambiamento per favorire la trasposizione di- dattica a distanza}

La flessibilità e l'adattamento al cambiamento, intese in questo articolo nei termini proposti secondo la declinazione didattica del lavoro di ricerca di Alain Berthoz sulla semplessità (Berthoz, 1997, 2003, 2009, 2011, 2015; Sibilio 2011, 2012a, 2012b, 2014a, 2014b, 2015), possono favorire la trasposizione didattica nel delicato passaggio dalle lezioni in presenza a quelle svolte attraverso la didattica a distanza. Difatti, esse si definiscono come quelle capacità didattiche dinamiche e flessibili (Sibilio e Zollo, 2016), che consentono di fronteggiare la complessità che ininterrottamente emerge da diversi fattori:

1) dalla rete di relazioni che collega i luoghi e i setting didattici (Ibidem);

2) i prodotti e i processi che emergono dalle pianificazioni didattiche (Laurillard, 2014);

3) dal sapere sapiente che diviene sapere insegnato (Nigris, 2014; Rossi e Pezzimenti, 2014); 
4) dall'interazione docente-discente (Ibidem). Assieme alle altre proprietà e principi della semplessità, la flessibilità e l'adattamento al cambiamento contribuiscono a costituire strumenti potenziali in grado di svolgere una funzione antagonista (Sibilio e Zollo, 2016) alla didattica tradizionale, frontale e lineare; la loro presenza garantisce sia dai rischi dell'egocentrismo dell'azione che dall'inefficacia di lettura e di interpretazione affidabile dei bisogni del discente consentendo ai docenti "di leggere la specificità del contesto, le richieste ed i vincoli individuali ed interindividuali del processo di insegnamento-apprendimento, adattandosi flessibilmente" (Ibidem). La flessibilità e l'adattamento al cambiamento, corrispondono alla capacità di agire con modalità diverse in rapporto al contesto, che in questo caso cambia radicalmente veicolandosi unicamente sui media digitali. La flessibilità è pertanto una delle proprietà semplesse che interpreta maggiormente

la funzione costruttivista e nondimeno enattiva della didattica, e corrisponde alla capacità dell'insegnamento di adattarsi al cambiamento in funzione del soggetto, delle sue caratteristiche, del contesto e della tipologia di apprendimento atteso. La flessibilità è quindi la capacità di decifrare la complessità dei bisogni formativi e adeguare costantemente l'insegnamento alla situazione e al soggetto che apprende; è una forma semplessa che coniuga una capacità diagnostica e una prognostica attraverso l'utilizzo di proprietà didattiche capaci di mettere in gioco, capitalizzare e far interagire le risorse del discente e quelle del docente. La flessibilità naturalmente può tradursi nella scelta di forme vicarianti dell'insegnamento, come quelle centrate sul movimento, sul gesto e sulla mimicasi ricordi a tal proposito quanto esposto riguardo al software Kinoni - che possono essere alternative $\mathrm{o}$ complementari ad altre modalità didattiche semplessemente adattive e necessariamente rispondenti ai significati che corpo e movimento possono assumere per il soggetto in ragione delle sue caratteristiche e dalla sua esperienza (Sibilio, 2014, p. 341).

Più nel dettaglio, il docente può mettere in atto, in modo flessibile, la trasposizione didattica attraverso due direttive:

1) la ricostruzione delle «discipline basilari affinché le idee fondamentali e più feconde - abbiano - un posto centrale» (Rossi e Pezzimenti, p.168);

2) l'adeguare in modo opportuno «le difficoltà dei contenuti di studio alle capacità degli alunni» (Ibidem) riorganizzando gli aspetti strutturali del sapere insegnato. Pertanto, si deve agire su entrambi i momenti che contraddistinguono la trasposizione didattica interna:

1) la cronogenesi «che corrisponde alla pianificazione o progettazione dei contenuti da insegnare» (Ibidem) che deve prevedere i possibili problemi che insorgeranno durante la didattica a distanza, ad esempio: disconnessioni degli utenti, perdita di segnale e di banda, strumenti vicarianti per fare lezioni asincrone, in cui entra in gioco l'anticipazione probabilistica (Berthoz, 2011, 2015; Sibilio 2014a, 2014b, 2015).; 
2) la topogenesi in cui si fa lezione, in cui emergono le problematiche.

Sicuramente, per una didattica a distanza di qualità è necessario prestare una maggior cura durante la fase di cronogenesi della trasposizione didattica fondata sui principi di insegnamento come scienza della progettazione (Laurillard, 2014) e di pedagogia intesa come scienza pratico-progettuale (Pellerey, 2011); avvalendosi di modalità di operare già mature e consolidate quali gli Episodi di Apprendimento Situato (Rivoltella, 2013, 2016). Per quanto riguarda la fase di topogenesi della trasposizione didattica, che risulta spesso dinamica e soggetta a imprevisti, la flessibilità e l'adattamento al cambiamento permettono concretamente di fronteggiare le differenti situazioni che emergono durante il corso delle lezioni per realizzare quegli «oggetti di insegnamento» (Rossi e Pezzimenti, p. 170) che sono "creati" e rimodulati di volta in volta dai docenti in base alla loro esperienza, alle circostanze e risultano comunque diversi dagli «oggetti del sapere» (Ibidem). Attraverso il principio di vigilanza epistemologica, espresso da Chevallard, avverrà, anche nella didattica a distanza, «una vera e propria 'responsabilità epistemologica' degli insegnanti nei confronti dell'oggetto di insegnamento» (Ibidem), tracciando la rotta e richiamando al principio semplesso del senso (Berthoz, 2011; Sibilio, 2014). Sintetizzando, l'anticipazione probabilistica porterà i suoi benefici alla fase di cronogenesi della trasposizione didattica e la flessibilità e l'adattamento al cambiamento permetteranno di poter orientarsi nella quotidianità del processo di insegnamento-apprendimento durante la topogenesi.

\section{Presentazione dei dati relativi alla didattica a distanza}

In questo paragrafo si riportano i risultati di un questionario, che aveva come tema il passaggio dalla didattica in presenza a quella a distanza, somministrato ai partecipanti al percorso formativo dei 24-CFU dell'Università degli Studi del Sannio di Benevento, del secondo semestre dell' anno accademico 2019/20, più nel dettaglio, agli studenti di "Tecnologie didattiche per l'inclusione" (SSD M-PED/03). Il questionario è stato compilato da centottantatré studenti laureandi o laureati (ottantadue donne e centouno uomini) tra i quali alcuni docenti precari; per raccogliere altri dati, il questionario è stato somministrato a cinquantadue docenti campani disciplinaristi e non di sostegno (trentasei donne e sedici uomini) che svolgono la loro funzione docente nelle scuole secondarie di secondo grado. La scelta di quest'ordine e di questo grado è motivata dal fatto che è il più prossimo alla formazione universitaria sia per età degli studenti e sia per tipologia di didattica.

Il numero totale di partecipanti è pertanto salito a duecentotrentacinque, di conseguenza i risultati qui riportati non hanno una validità statistica tale da ef- 
fettuare inferenze su larga scala; tuttavia possono svolgere la funzione di caleidoscopio su una realtà specifica ovvero quella della provincia di Benevento e dei territori limitrofi. Lo scopo del questionario è stato quello di poter verificare se gli intervistati, nella loro esperienza di didattica a distanza, hanno effettivamente beneficiato di una serie di «modalità diffuse di apprendimento - in rete caratterizzate - da multitasking, autorialità e socialità» (Falcinelli, 2014, p.89).

Entrando nel merito della modalità di compilazione del questionario, ecco le principali caratteristiche: 1) sono state presentate delle asserzioni ed è chiesto all'intervistato quanto fosse d'accordo con esse in una scala a cinque valori (tranne per i primi tre items e gli ultimi due) che andava da "per niente d'accordo" a "completamente d'accordo"; 2) era anonimo; 3) non era obbligatorio rispondere a tutte le domande; 4) le domande erano strettamente correlate tra loro per esplorare il tema della didattica a distanza da varie prospettive; 5) per verificare il grado di affidabilità, sono state inserite delle domande "civetta", non riportate in questo articolo, che servivano a verificare la coerenza dalla compilazione, grazie a tale procedimento è stato possibile dedure che nel caso pessimo i questionari compilati con superficialità non superano il $16,18 \%$; 6) per coloro che insegnavano sono state richieste informazioni aggiuntive relative a quanto avvenuto, durante il passaggio della didattica in presenza a quella a distanza, ai loro studenti con bisogni educativi speciali; 7) prima della somministrazione, è stato ripetuto più volte, a chi partecipava alla ricerca, di sentirsi libero di rispondere genuinamente; 8) è stato realizzato con Google Moduli; 9) in modo non esplicito, attraverso la lettura degli items stessi, all'intervistato era proposta un'analisi SWOT (Strengths, Weaknesses, Opportunities, and Threats), pertanto si alternavano domande legate alle opportunità, ai punti di forza, di debolezza e ai rischi della didattica a distanza.

Procedendo ora alla presentazione dei singoli items, in primis è stato chiesto, a chi ha compilato il questionario, quale tecnologia ha preferito utilizzare, come discente, nella didattica a distanza (potendone indicare pure più). Di seguito la figura 1 riporta tali informazioni.

Fig. 1 - Le tecnologie che gli intervistati hanno preferito utilizzare, come discenti, per la didattica a distanza durante l'emergenza sanitaria (si poteva indicare più di una risposta)

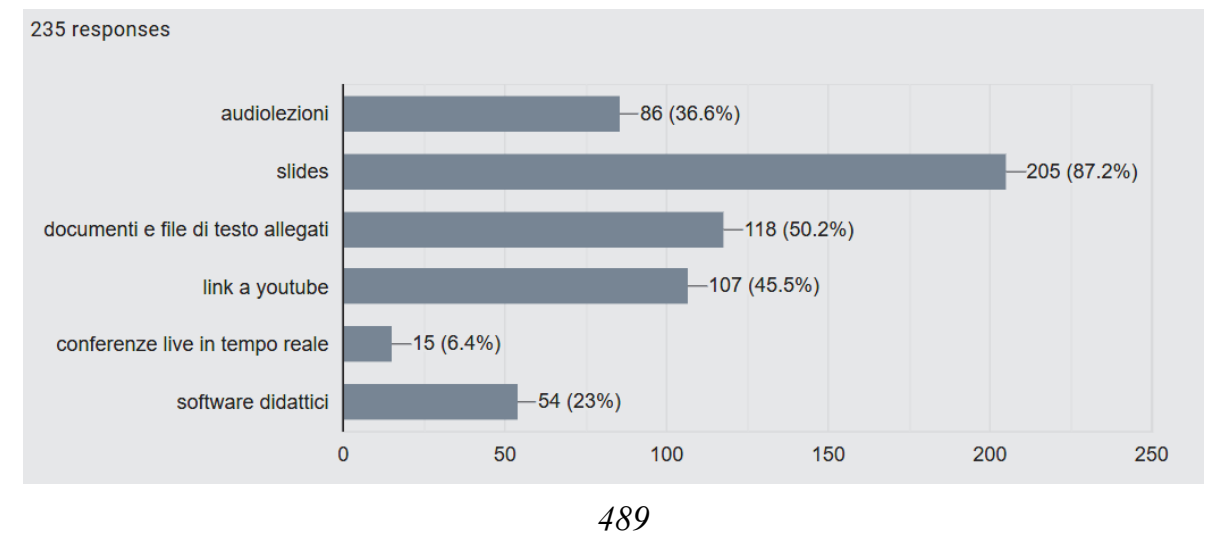


Nell'istogramma precedente, gli studenti e i docenti hanno riportato le tecnologie utilizzate nella didattica a distanza sia nel corso di "Tecnologie didattiche per l'inclusione" dei 24-CFU sia in altri corsi. Per quanti riguarda il corso appena menzionato, sono state utilizzate tutte le tecnologie elencate tranne le conferenze live. Nel secondo item è stato richiesto agli intervistati di elencare cosa preferiranno utilizzare in futuro da discenti. In tal modo è stato possibile conoscere l'incremento o il decremento, per ogni tecnologia presa in esame e poter effettuare delle prime valutazioni relative al differenziale tra questo item e il precedente per poterne trarne alcune conclusioni.

Fig. 2 - Le tecnologie che gli intervistati preferiranno utilizzare in futuro, da discenti, per la didattica a distanza (si poteva indicare più di una risposta)

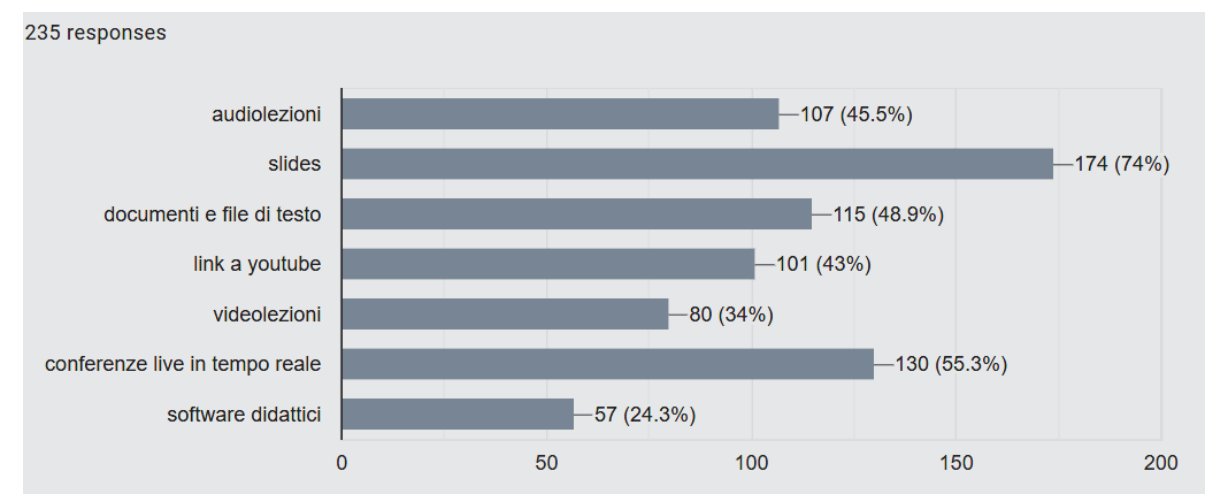

È emerso che l'interesse per le audiolezioni ha avuto un incremento del $8,9 \%$, le slides, come strumento a supporto delle lezioni, hanno avuto un decremento del $13,2 \%$, l'interesse per i documenti di testo a supporto della didattica invece non hanno registrato particolari variazioni, come pure i video proposti dal docente attraverso link di YouTube. Purtroppo, le videolezioni preregistrate non hanno un termine di paragone perché non erano state usate nel corso di "Tecnologie didattiche per l'inclusione". Per quanto riguarda i software didattici, da intendersi pure come piattaforme quali Edmodo, WeSchool, Google Classroom, etc. non hanno subito particolari variazioni. Si potrebbe ipotizzare, che in funzione della flessibilità e adattamento al cambiamento dovuto all'emergenza sanitaria e alla migrazione dalla presenza alla didattica a distanza l'interesse storicamente riservato alle slides si è riposizionato sui contenuti audio offerti dal docente. Quindi è stato posto il focus sempre sul futuro, richiedendo però agli intervistati cosa preferiranno utilizzare come docenti, nella loro didattica a distanza, i risultati sono variati parecchio; difatti, un conto è usufruire di una tecnologia, diverso è quando si devono preparare artefatti digitali 
e learning object (Rivoltella, 2013, 2014, 2015) oppure amministrare piattaforme di e-learning.

Fig. 3 - Le tecnologie che gli intervistati preferiranno utilizzare il futuro, da docenti, per la didattica a distanza (si poteva indicare più di una risposta)

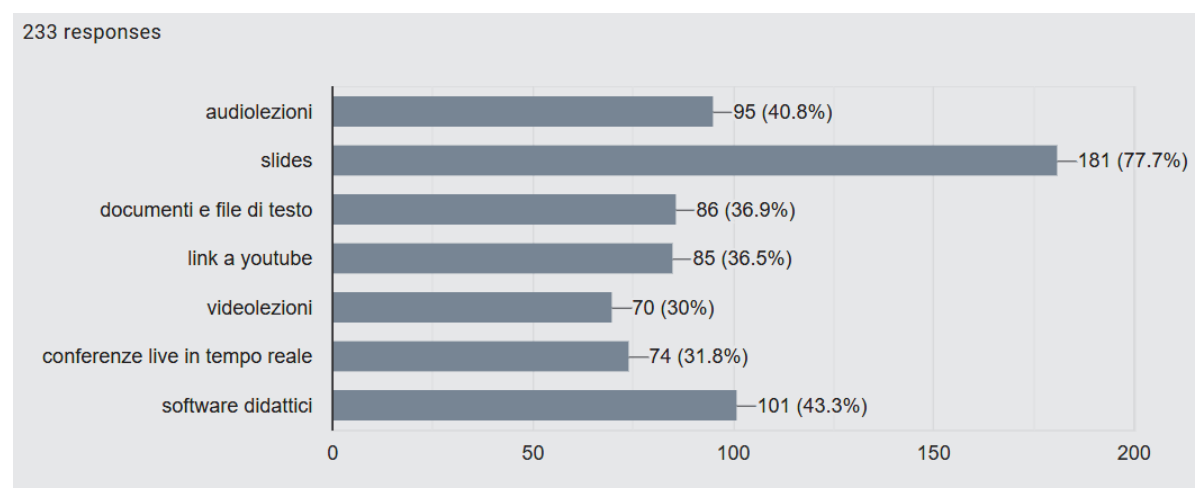

Purtroppo, l'idea principale degli intervistati è quella di realizzare slides, $77,7 \%$, e solo il $43 \%$ si affiderà a piattaforme on-line per scopi didattici (se non obbligati dalla propria istituzione scolastica). Il desiderio predominante è quello di trovare soluzioni rapide e non sincrone, solo il $31,8 \%$ ipotizza soluzioni in tempo reale. In pochi si dedicheranno alla realizzazione soluzioni a più alto contenuto tecnologico quali artefatti digitali e learning object audio o audiovideo. Nell'item successivo, è stato chiesto agli intervistati quanto fossero d'accordo con la seguente affermazione: il docente, nella didattica a distanza, deve tenere in considerazione tutte le funzioni dell'accoglienza proponendo contenuti e orientando gli studenti «attraverso il modeling, fornendo feedback, e commentando e valutando le attività svolte» (Rivoltella, 2020).

Fig. 4 - Relazione tra il concetto di accoglienza e quello di didattica a distanza

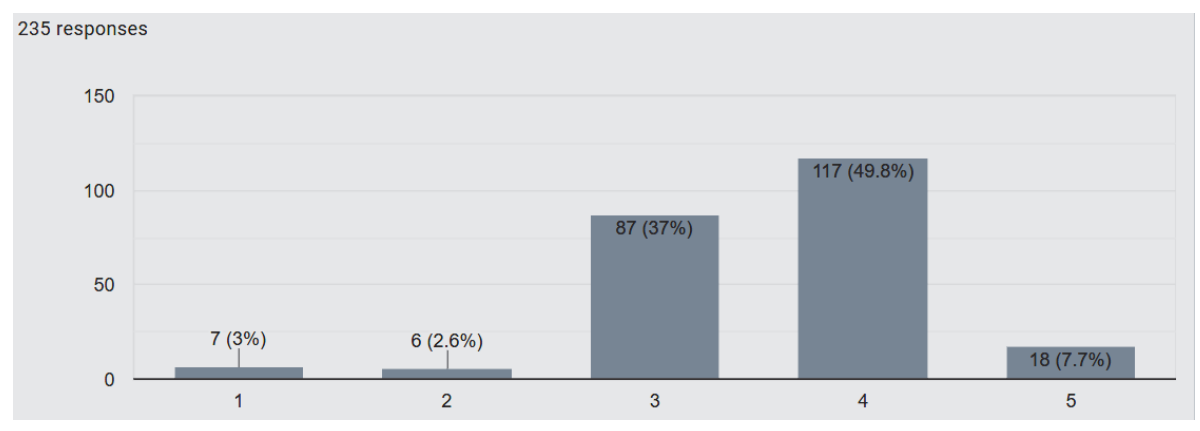


In media, gli studenti erano d'accordo con quanto affermato nell'item. In seguito, è stato chiesto di esprimere la propria opinione riguardo il concetto di attività, così sintetizzato: «è bene attivare gli studenti proponendo lavori» (brevi esercizi, analisi di casi, ricerche, elaborazione di progetti) «individuali o di gruppo in linea con le caratteristiche e necessità della disciplina» (Ibidem). Di seguito i risultati emersi.

Fig. 5 - Relazione tra il concetto di attività e quello di didattica a distanza

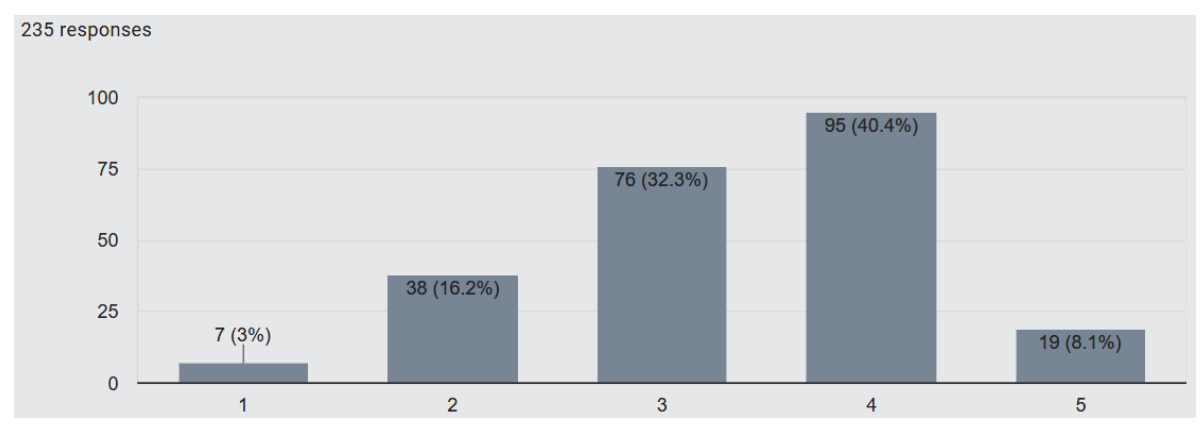

Mediamente, gli studenti erano abbastanza d'accordo. Si noti che per quanto riguarda i centottantatré corsisti, essi avevano avuto modo di fare l'esperienza sopra descritta, nel corso dei 24-CFU, approfondimenti e percorsi virtuali realizzati ad-hoc, analisi di casi, ricerche e webquest che avevano come oggetto la didattica outdoor, la didattica museale, il processo di insegnamento formale, non-formale e informale (Pellerey, 2002, 2011) posto in relazione con le tecnologie didattiche, la flexible classroom (Todino, Aiello e Sibilio, 2016) e il seamless learning (Magnoler, Tiraboschi e Trentin, 2019; Trentin, 2019; Seow, Boticki e Chia, 2019; Beozzo e Colombo, 2019; Bubbio, Candeo e La Cava, 2019). Nell'item seguente è stato chiesto agli intervistati di ragionare sulla seguente proposizione: c'è un rischio, hai tenuto conto che «finita l'emergenza si torni alla normalità: la vecchia didattica trasmissiva» (Rivoltella, 2020). Di seguito i risultati.

Fig. 6 - Relazione tra il concetto di ritorno alla normalità post emergenza sanitaria e quello di didattica a distanza

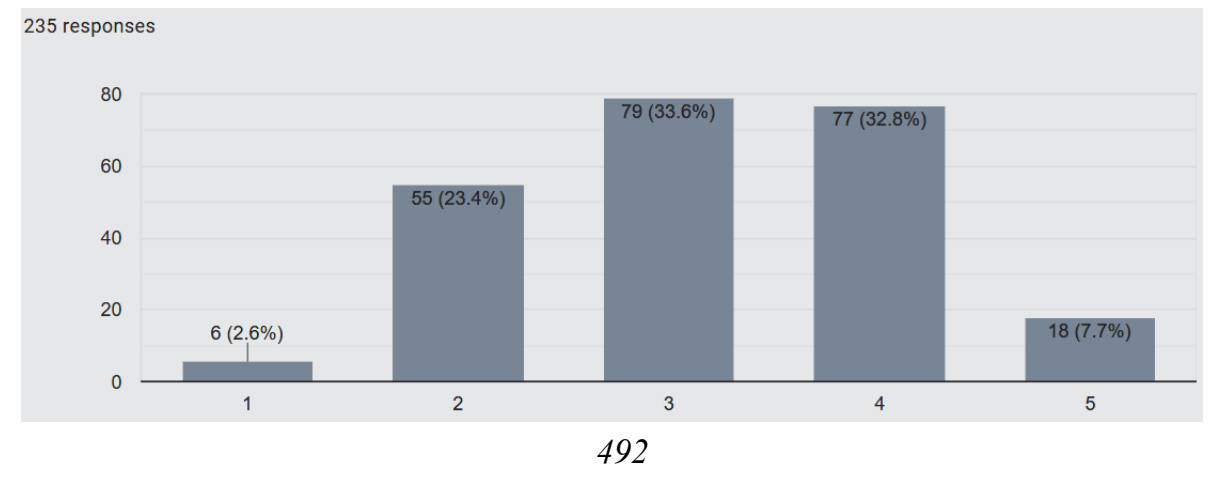


Su questo item il gruppo si è spaccato, in modo evidente, in quattro sottogruppi. Si può supporre che, ogni individuo è forse influenzato dalla propria percezione del fenomeno in esame, dal proprio Umwelt (Berthoz, 2011; Aiello, et al., 2013; Sibilio, 2014a, 2014b) e dalla propria Weltanschauung che a volte è più incline, a volte meno, all 'adattamento al cambiamento imposto da fattori esterni e non da scelte personali. Pertanto, com'era prevedibile, alcuni docenti, finita l'emergenza sanitaria, preferiranno tornare alla vecchia didattica trasmissiva e abbandoneranno le tecnologie.

Nell'item successivo è stata posta l'attenzione sulla frase: «serve pensare che la qualità della relazione non sia una questione (...) di strumenti e che il digitale non è un'alternativa alla presenza ma una sua dimensione» (Rivoltella, 2020). Di seguito i dati raccolti.

Fig. 7 - Attinenza tra il concetto di qualità della relazione docente-discente e quello di didattica a distanza

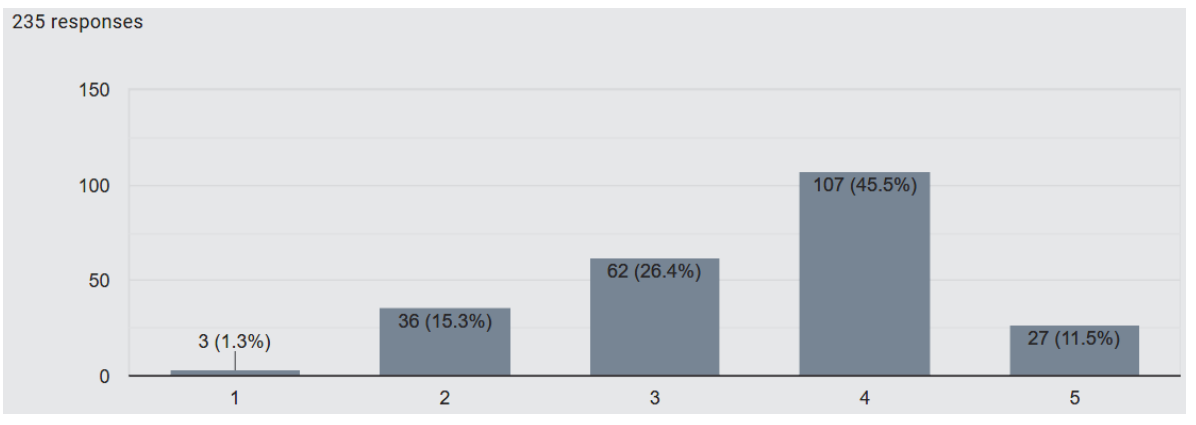

In questo caso il gruppo si è ricompattato affermando per il $45,5 \%$ di essere "molto d'accordo" al concetto messo in risalto dall'asserzione precedente. Nel prossimo item, scavando ancora sulla questione della relazione docente-discente, la frase proposta è stata: «la relazione è il risultato dell'intenzionalità educativa, è la consapevolezza che l'altro è al centro della mia attenzione e il digitale può essere uno dei modi per mantenercelo» (Ibidem). Ecco i dati raccolti.

Fig. 8 - Attinenza tra il concetto di consapevolezza dell'altro e quello di didattica a distanza

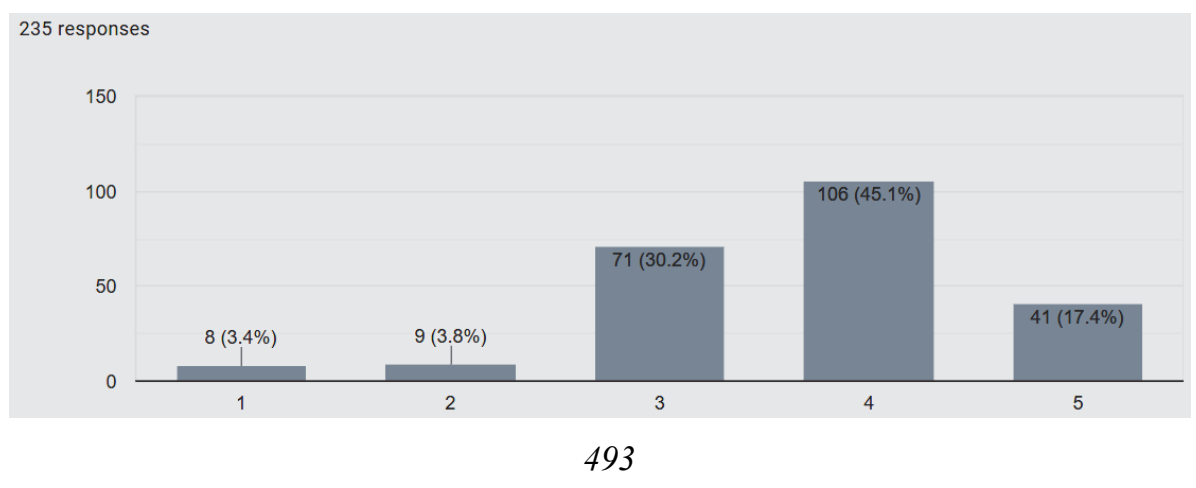


Anche in riferimento a questa domanda, coloro che hanno risposto sono compatti con quanto affermato da Rivoltella. Nell'item successivo, incalzando delle relazioni sul tema è stato proposto di riflettere riguardo la seguente affermazione: «Il digitale può divenire carezza nei momenti di sconforto, supporto nei momenti di difficoltà, legame nelle situazioni di solitudine, presenza quando si sperimenta l'assenza» (Ibidem). Di seguito il relativo istogramma.

Fig. 9 - Attinenza tra il concetto di solitudine e quello di didattica a distanza

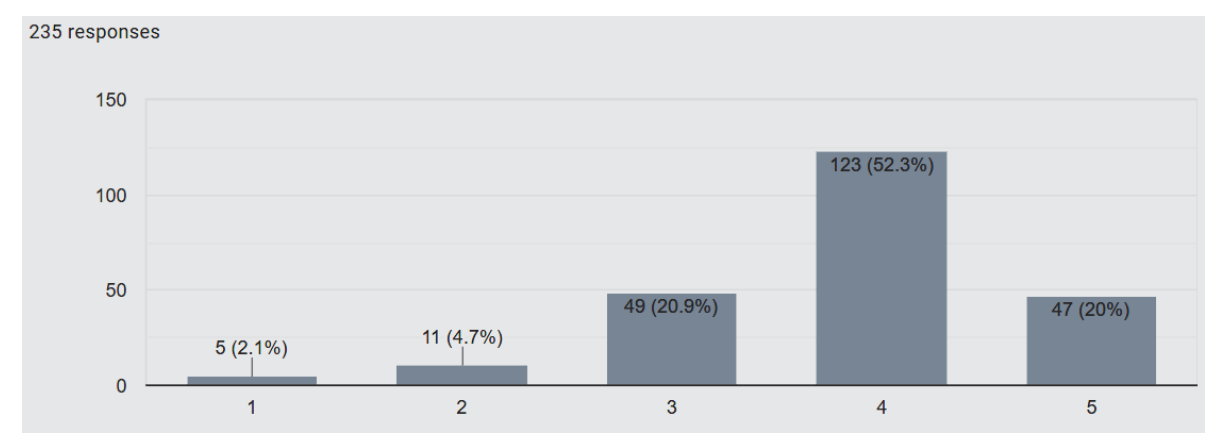

Il gruppo risultava compatto nel concordare di essere abbastanza d'accordo con la precedente affermazione. Una volta terminati gli items riferiti a queste riflessioni concernenti i concetti di relazione e solitudine, nell'item successivo è stata proposta una riflessione relativa al processo di insegnamento-apprendimento on-line di tipo sincrono, tale riflessione è stata riportata come segue: «Non basta 'mandare in onda' la lezione e continuare a parlare come si sarebbe fatto in aula. Va studiata una sceneggiatura» (Ibidem). Ecco di seguito le risposte.

Fig. 10 - Attinenza tra il concetto di motivazione e quello di didattica a distanza

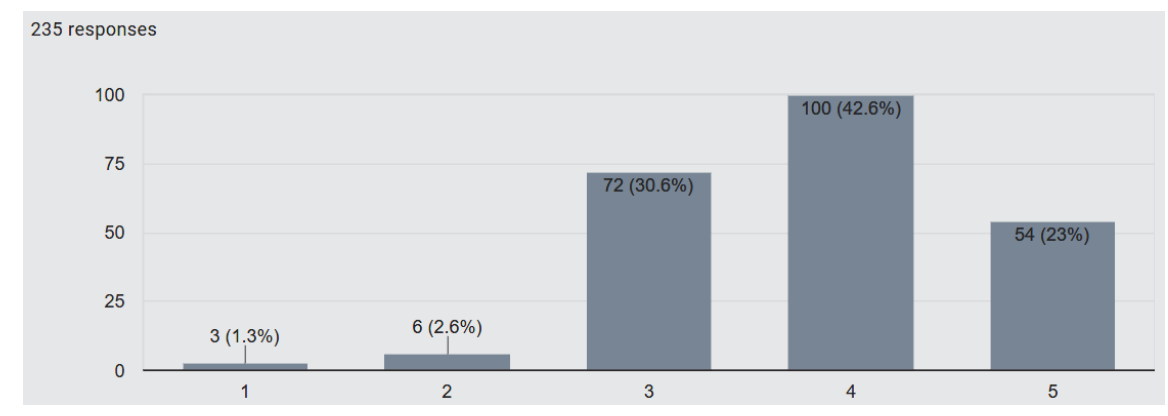

Dal grafico si evince una diversa sensibilità all'adattamento al cambiamento. Per il $23 \%$ è assolutamente necessario cambiare la struttura delle lezioni 
nella didattica a distanza. Questo risultato si può mettere in relazione al differenziale emerso in precedenza a proposito delle tecnologie che gli intervistati vorrebbero utilizzare come futuri docenti e quelle da usare come futuri discenti. Il questionario approfondisce quest'argomento attraverso un item che propone la seguente frase su cui riflettere: «Non basta più educare lo spettatore [delle videolezioni], occorre anche educare il produttore [delle lezioni] questo significa che insieme al pensiero critico occorre sviluppare anche la responsabilità» in termini di Media Education (Ibidem). Di seguito l'istogramma.

Fig. 11 - Attinenza tra il concetto di pensiero critico (in termini di Media Education) e quello di didattica a distanza

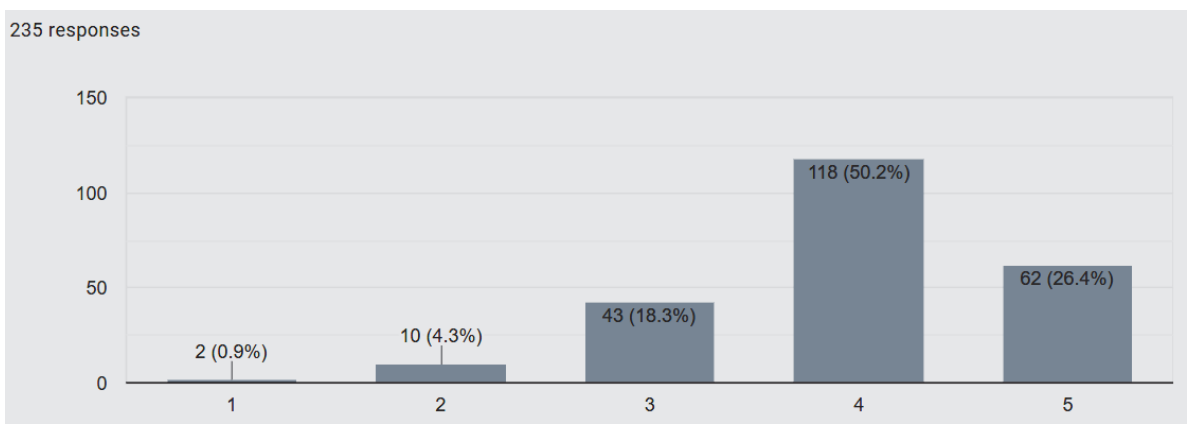

$\mathrm{Su}$ quest'argomento il gruppo è compatto nell'affermare di essere molto d'accordo (oltre il 50\%) o completamente d'accordo (oltre il $26 \%$ ), forse questo raggruppamento sui valori massimi è dovuto al fatto che l'affermazione pone l'intervistato in una posizione eterocentrica (Berthoz, 2011) rispetto al fenomeno descritto, in altre parole non pone chi risponde, in modo diretto, né nei panni del docente né in quelli del discente. Poi il focus del questionario vira su un altro tema didattico d'attualità: «la gestione dell'informazione al tempo del Covid-19 ci conferma l'esigenza di Information Literacy (accuratezza delle fonti etc.)» (Rivoltella, 2020). Di seguito i valori delle risposte.

Fig. 12 - Attinenza tra il concetto di Information Literacy e quello di didattica a distanza

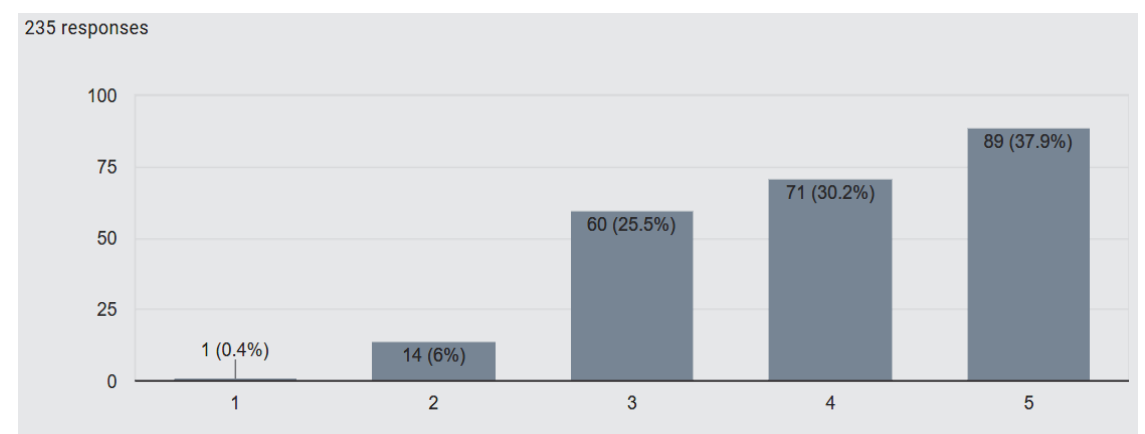

Gli intervistati sono particolarmente sensibili all'Information Literacy, in 
effetti, questo può essere motivato dal fatto che erano tutti laureandi, laureati o docenti. L'item successivo invece riguardava il diritto alla riservatezza delle informazioni personali e in generale il tema della privacy nei sistemi e nelle piattaforme di distance education ed era chiesto loro quanto quest'argomento li preoccupava. Di seguito le risposte.

Fig. 13 - Attinenza tra il concetto di privacy e la didattica a distanza

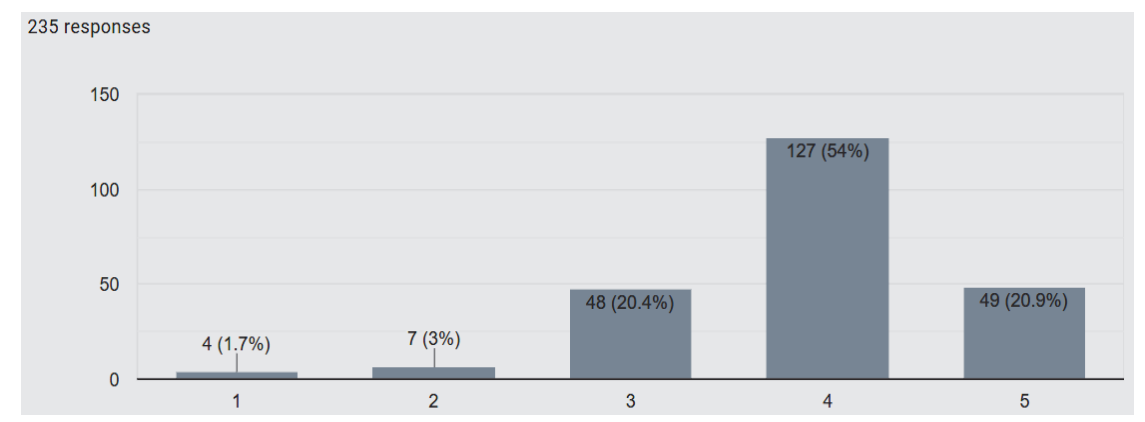

Probabilmente per un senso pratico, per flessibilità e adattamento al cambiamento e alle necessità oggettive dell'emergenza sanitaria, la sicurezza informatica diviene un fattore cruciale nella didattica a distanza, perché non solo si lega al diritto alla riservatezza delle informazioni personali ma anche della propria vita privata. L'item successivo del questionario è stato posto solamente a chi era insegnante (precario o di ruolo), chiedendo esplicitamente agli intervistati se erano stati esclusi dalle classi virtuali, attivate nell'istituto nel quale prestavano servizio, alcuni dei loro studenti, per raccogliere dati riguardanti il concetto d'inclusione scolastica (Cottini, 2008, 2017; Fiorucci, 2014; Giaconi, 2014; Mura, 2016; Dovigo, 2017; D’Alonzo 2017). Ogni docente poteva indicare pure più di una situazione di esclusione dalle classi virtuali attivate nelle loro scuole. Di seguito le risposte a tale item.

Fig. 14 - Il problema dell'esclusione scolastica degli alunni con Bisogni Educativi Speciali emersa con la didattica a distanza

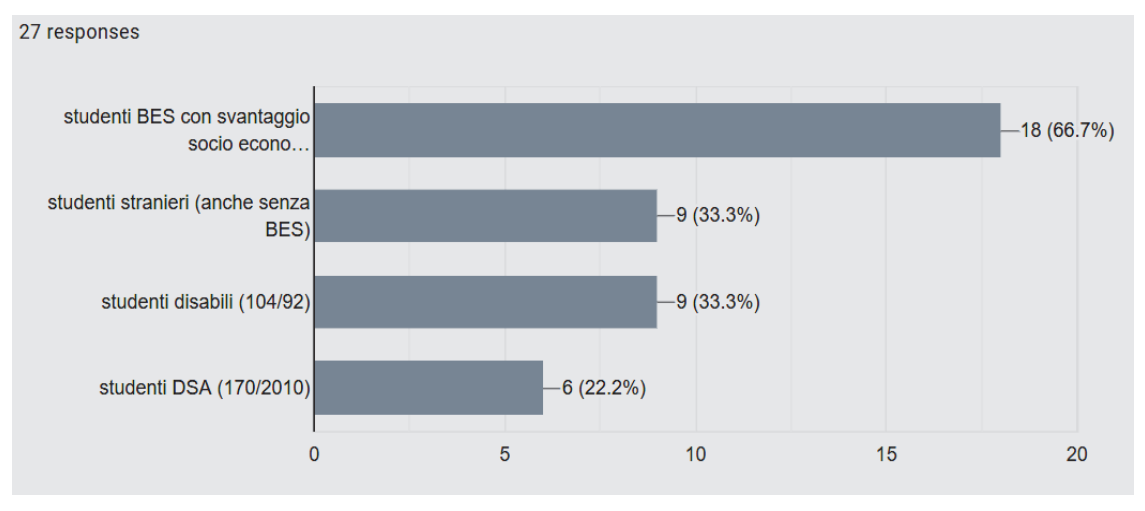

496 
Questo item si deve leggere come segue: i casi di esclusione, noti al sottogruppo di docenti intervistati, erano ventisette; tuttavia, non è possibile conoscere, per anonimato, se tali casi erano avvenuti nella stessa scuola e quanti docenti abbiano vissuto questa situazione in prima persona.

L'ultima domanda, rivolta a tutti gli intervistati, esaminava ancora l'inclusione scolastica e riguardava il superamento del Digital Divide, che a maggior ragione ha colpito gli studenti con svantaggi economici. Per risolvere questo problema era stato chiesto di indicare una soluzione tra due proposte: 1) un "bonus studenti" per l'acquisto del dispositivo per favorire il BYOD (Bring Your Own Device); 2) il comodato d'uso offerto dalla scuola allo studente, e pertanto l'owner del device era scuola e non lo studente. Di seguito le risposte.

Fig. 15 - Possibile soluzione (solo da un punto di vista di strumentazione tecnologica) dell'esclusione scolastica degli studenti con Bisogni Educativi Speciali durante il passaggio dalla didattica in presenza alla didattica a distanza

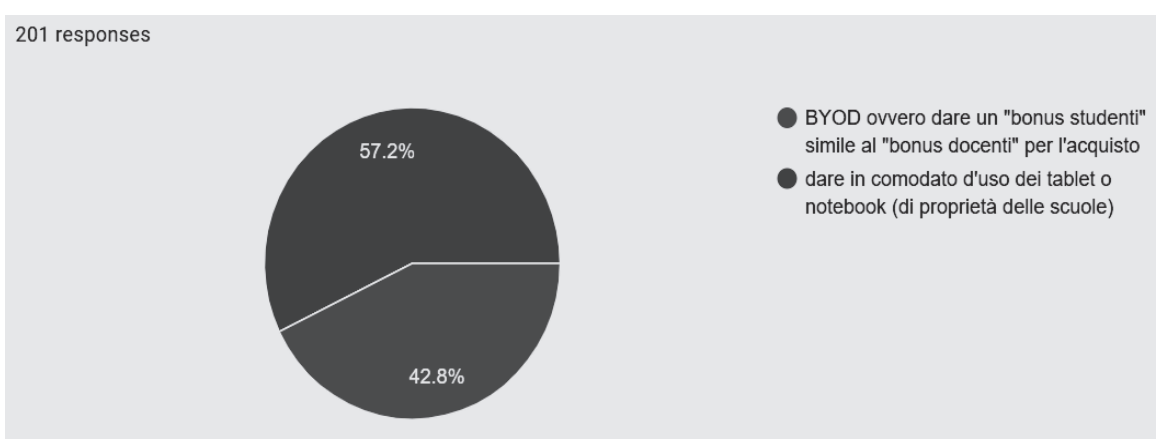

Tra gli intervistati si percepisce una leggera inclinazione nei confronti del BYOD forse dovuto al fatto che il comodato d'uso provoca degli immobilizzi che si svalutano rapidamente e che alla scuola effettivamente generano una situazione anti-economica, ma questa è solo una supposizione.

\section{Riflessioni conclusive}

La pluralità di studi che anima la ricerca in ambito educativo, offre ampie possibilità di indagine e di riflessione sulla professionalità docente e sulla centralità del suo agire che non costituisce solo la modalità di cui dispone per facilitare l'edificazione dei saperi ma anche la trasposizione di un'intenzionalità che non è mai avulsa da un radicato sistema di valori, credenze, atteggiamenti naturalmente connessi alla propria soggettività; una complessità della funzione docente che «quando si riferisce nello specifico al come insegnare, evidenzia la dimensione qualitativa e quantitativa che caratterizza lo spazio delle sue scelte 
e delle sue azioni intenzionali e quindi necessita della piena consapevolezza dei confini del proprio agire» (Sibilio, 2011, p. 50).

Le azioni didattiche diventano, dunque, l'esito di un consapevole processo decisionale in funzione di uno scopo: per il docente «il "dopo" viene anticipato mentalmente, come desiderio e come calcolo, mentre il "prima" assolve la funzione di problema alla luce di quanto vuole e può fare in modo che accada "dopo". Più che un processo, egli ha dinanzi un "progetto"» (Damiano, 1999, p.86). La sua si caratterizza come una progettualità didattica in continua ridefinizione, negli obiettivi, nei contenuti, nelle metodologie, negli strumenti, sulla base di variabili interne ed esterne alla relazione educativa: il docente "non pratica un laboratorio dove tutto procede sotto controllo. Al contrario, opera in una situazione di contingenze, dove è tenuto ad intervenire in pubblico, senza rete, in tempo reale, in situazioni incerte, di volta in volta da decifrare e interpretare» (Damiano, 2013, p. 290). Rispetto a queste continue, veloci e inaspettate trasformazioni è chiamato a rinnovarsi costantemente attraverso l'esercizio della propria agentività individuale:

In questa sfida adattiva vengono premiate le persone che si sentono capaci di determinare il proprio futuro. Molte teorie contemporanee dipingono l'individuo come uno spettatore passivo dei meccanismi interiori orchestrati dai fattori ambientali; le persone sarebbero prive di ogni senso di agentività. Al contrario, nella mia concezione, l'uomo è un organismo ambizioso capace di agire d'anticipo in previsione di problemi, necessità e cambiamenti, e attivo nel modellare il corso della sua vita e i sistemi sociali che organizzano, guidano e regolano le materie sociali (Bandura, 2000, p. 1).

L'agire didattico diventa, dunque, espressione tangibile delle capacità organizzative e proattive del docente che sceglie, progetta e pianifica, previene, anticipa e prospetta nuovi orizzonti esperienziali e conoscitivi per i suoi studenti dimostrando capacità di flessibilità ed un adattamento al cambiamento anche quando tali "contingenze" richiedono un inaspettato passaggio a nuovi spazi e tempi della didattica; ma anche quando un evento sembrerebbe essere d'ostacolo «diventa l'elemento pivot attorno a cui si articola la trasformazione del sistema. L'evento non annulla il ruolo del professionista, ma anzi lo esalta in quanto un atteggiamento scientifico richiede oggi la capacità di leggere il contesto, di operare in situazione» (Rossi, 2011, p. 146). L'azione trasformativa del docente, in questo percorso di costruzione della propria ed altrui conoscenza, è connotata da una sua naturale propensione a percepire, esperire, risolvere problemi e da un'innata vicarianza che «è sempre fondata su un atto, un'intenzione, uno scopo condiviso - ed è in grado di favorire - la creazione di nuove soluzioni» (Berthoz, 2015, p. 144; Sibilio, 2017).

L'esperienza svolta presso l'Università degli Studi del Sannio di Benevento ha richiesto ai docenti flessibilità e adattamento al cambiamento, la capacità di 
agire con modalità diverse, di adeguare il proprio insegnamento alle mutate esigenze dei discenti, ai nuovi spazi virtuali e ai nuovi luoghi divenuti piattaforme digitali. Dai dati del questionario somministrato ai centottantatrè studenti del corso di "Tecnologie didattiche per l'inclusione", ed esteso anche a cinquantaque docenti campani disciplinaristi e non di sostegno, che svolgono la loro funzione docente nelle scuole secondarie di secondo grado, è emerso, in linea con le affermazione di Rivoltella (2020) la necessità di una rivisitazione della didattica che richiede, seppure a distanza, una progettazione che tenga conto delle funzioni dell'accoglienza, della qualità della relazione basata sulla centralità dell'altro, di una trasposizione didattica che non si traduca in una mera diffusione di contenuti in rete, ma sia in grado di accompagnare e motivare il discente nel suo graduale processo di apprendimento attraverso azioni di modeling, feedback, commenti e valutazioni delle attività individuali o di gruppo. Nello stesso tempo, il rilevare che solo una parte degli intervistati realizzerà in futuro soluzioni a più alto contenuto tecnologico, quali artefatti digitali e learning object audio o audio-video, preferendo il modello della didattica trasmissiva, eventualmente arricchita da slide, e soluzioni didattiche rapide e asincrone, sollecita una riflessione circa l'importanza di incentivare la Media Education e l'Information Tecnology nei progetti di formazione docente anche in itinere.

In conclusione, la convergenza di approcci teorici e il superamento di antinomie e di rigidità disciplinari, ci restituisce una visione complessa, aperta e flessibile del ruolo del docente e della didattica che in quanto "cantiere aperto e operoso" (Perla, 2017, p. 169) è, dunque, pronta a continue ridefinizioni ed è capace di proporre, da un lato «un ampio repertorio di dispositivi dal quale-l'insegnante -sceglie quello più appropriato in base al contesto e alle finalità individuate. Dall'altro suggerisce i processi che permettono al docente di riflettere sul proprio operato, di esplicitare il proprio sapere e di mettere a punto il processo trasformativo» (Rossi, 2011, p. 147).

\section{Riferimenti bibliografici}

Aiello P., Sibilio M. (2013). Didactics and "Simplexity": Umwelt as a Perceptive Interface. Education Sciences \& Society, 27-35.

Aiello P., Sharma U., Sibilio M. (2016). La centralità delle percezioni del docente nell'agire didattico inclusivo: perché una formazione docente in chiave semplessa? Italian Journal of Educational Research, IX: 17-28.

Argyle M. (1992). Il corpo e il suo linguaggio. Studio sulla comunicazione non verbale. Bologna: Zanichelli.

Bandura A. (2000). Autoefficacia. Teoria e applicazioni. Trento: Erickson. 
Beozzo M., Colombo M. (2019). Nuovi paradigmi formativi per una nuova integrazione tra scuola e lavoro: sviluppare la cultura dell'apprendistato attraverso il seamless learning. Professionalità Studi, 4, Edizioni Studium, La Scuola: Brescia, pp. 51-68.

Berthoz A. (1997). Le sens du mouvement. Paris: Odile Jacob.

Berthoz A. (1998). Il senso del movimento. Milano: McGraw-Hill.

Berthoz A. (2003). La scienza della decisione. Torino: Codice.

Berthoz A. (2009). The human brain "projects" upon the world, simplifying principles and rules for perception. In: Berthoz A (Ed.). Neurobiology of "Umwelt": How Living Beings Perceive the World. Guilford: Springer London Boulder, pp. 17-27.

Berthoz A. (2011). La semplessità. Torino: Codice.

Berthoz A. (2015). La vicarianza. Il nostro cervello creatore di mondi. Torino: Codice.

Bocchi G, Ceruti M. (1985). La sfida della complessità. Milano: Feltrinelli.

Borghi A. M., Iachini T. (2004). Scienze della mente. Bologna: Il Mulino.

Bubbio R., Candeo S., La Cava M. (2019). Seamless learning nelle organizzazioni: utopia o realtà? Un viaggio tra neuroscienze ed ecosistemi formativi per comprendere le sfide delle aziende 4.0. Professionalità Studi, 4, Edizioni Studium, La Scuola: Brescia, pp.88-117.

Caruana F., Borghi A. (2016). Il cervello in azione. Introduzione alle nuove scienze della mente. Bologna: il Mulino.

Ceruti M. (2000). Il vincolo e la possibilità. Milano: Feltrinelli.

Corsi M., Stramaglia M., Gellel A.M. (2019). Giovani, studenti e infiniti mondi. Education Sciences \& Society, S. 1., 10(2): 7-11.

Cottini L. (2008). Per una didattica speciale di qualità: dalla conoscenza del deficit all'intervento inclusivo. Perugia: Molacchi.

Cottini L. (2017). Didattica speciale e inclusione scolastica. Roma: Carocci.

Crispiani, P. (2008). Un lavoro per la didattica. In: Giaconi, C. Le vie del costruttivismo. Roma: Armando Editore.

D’Alonzo L. (2017). La differenziazione didattica per l'inclusione. Metodi, strategie, attività. Trento: Erickson.

Damiano E. (1999). L'azione didattica: per una teoria dell'insegnamento. Roma: Armando Editore.

Damiano E. (2008). Costruttivismo. Aria di famiglia, premesse epistemologiche e prospettive didattiche. In: Giaconi C. (2008). Le vie del costruttivismo. Roma: Armando Editore.

Damiano E. (2013). La mediazione didattica. Per una teoria dell'insegnamento. Milano: FrancoAngeli.

Durand M., Poizat G. (2017). Enazione, attività umana e ambienti di formazione. In: Rossi P.G. e Rivoltella P.C., a cura di, L'agire didattico. Brescia: La Scuola.

Fabbri L. (2014). I sistemi formativi fra teorie in uso e teorie dichiarate. In: Rossi P.G. e Rivoltella P.C., a cura di, L'agire didattico. Brescia: La Scuola.

Falcinelli F. (2014). Le tecnologie dell'educazione. In: Rossi P.G. e Rivoltella P.C., a cura di, L'agire didattico. Brescia: La Scuola. 
Fiorucci A. (2014). Gli atteggiamenti degli insegnanti verso l'inclusione e la disabilità: uno sguardo internazionale. Italian Journal of Special Education for Inclusion, 2(1): 53-66.

Frauenfelder E. (1983). La prospettiva educativa tra biologia e cultura. Napoli: Liguori.

Fraunfelder E. (1994). Pedagogia e biologia. Una possibile alleanza. Napoli: Liguori.

Gazzaniga M. S. (1990). Stati della mente. Stati del cervello. Come l'interazione di mente e cervello crea la nostra vita cosciente. Firenze: Giunti.

Gazzaniga M. S. (1999). La mente inventata. Le basi biologiche dell'identità e della coscienza. Milano: Guerini e Associati.

Gazzaniga M. S. (2007). L'interprete. Come il cervello decodifica il mondo. Roma: Di Renzo Editore.

Gennari M. (2006). Istituzioni di didattica. In: Giaconi M., a cura di, Didattica generale. Milano: Bompiani, pp. 29-78.

Giaconi C. (2014). Co-progettare l'inclusione. Milano: FrancoAngeli.

Giaconi C. (2008). Le vie del costruttivismo. Roma: Armando Editore.

Hall E.T. (1969). Il linguaggio silenzioso. Milano: Garzanti.

Johnson M. (2017). Embodied Mind, Meaning, and Reason: How Our Bodies Give Rise to Understanding. Chicago: The University of Chicago Press.

Lakoff G., Johnson M. (1999). Philosophy in the Flesh: the Embodied Mind \& its Challenge to Western Thought. New York: Basic Book.

Laurillard D. (2014). Insegnamento come scienza della progettazione: costruire modelli pedagogici per apprendere con le tecnologie. Milano: FrancoAngeli.

Magnoler P., Tiraboschi T., Trentin G. (2019). Saperi, apprendimenti e orientamento: uno sguardo d'insieme per avvicinarsi al seamless learning. Professionalità Studi, 4, Edizioni Studium, La Scuola: Brescia, pp.1-7.

Maturana H.,Varela F. (1985). Autopoiesi e cognizione. Venezia: Marsilio Editore.

Maturana H., Varela F. (1999). L'albero della conoscenza. Milano: Garzanti.

Miller J. G. (1986). La teoria generale dei sistemi viventi. Milano: FrancoAngeli.

Morin E. (1993). Introduzione al pensiero complesso. Gli strumenti per affrontare la sfida della complessità. Milano: Sperling \& Kupfer.

Morin E. (1995). Il metodo. Ordine, disordine, organizzazione. Milano: Feltrinelli.

Morin E. (2000). La testa ben fatta. Riforma dell'insegnamento e riforma del pensiero. Milano: Raffaello Cortina Editore.

Mura A. (2016). Diversità e inclusione. Prospettive di cittadinanza tra processi storicoculturali e questioni aperte. Milano: FrancoAngeli.

Nigris E. (2014). Didattica e saperi disciplinari: un dialogo da costruire. In: Rossi P.G. e Rivoltella P.C., a cura di, L'agire didattico. Brescia: La Scuola.

Oliverio A. (2017). Il cervello che impara. Neuropedagogia dall'infanzia alla vecchiaia. Firenze: Giunti.

Orefice P., Sarracino V., a cura di (2006). Cinquant'anni di pedagogia a Napoli: studi in onore di Elisa Frauenfelder. Napoli: Liguori.

Pellerey R. (2002). Didattiche dell'extrascolastico. In: Gennari M., a cura di, Didattica generale. Milano: Bompiani. 
Pellerey R. (2011). Educare. Per una pedagogia intesa come scienza pratico-progettuale. Roma: LAS.

Perla L. (2014). Teorie e modelli. In: Rossi P.G. e Rivoltella P.C., a cura di, L'agire didattico. Brescia: La Scuola.

Rivoltella P. C. (2012). Neurodidattica. Insegnare al cervello che apprende. Milano: Raffaello Cortina Editore.

Rivoltella P.C., Rossi P.G., a cura di (2012). L'agire didattico. Manuale per l'insegnante. Brescia: La Scuola.

Rivoltella P.C. (2013). Fare didattica con gli EAS. Brescia: La Scuola.

Rivoltella P.C. (2014). La previsione. Neuroscienze, apprendimento e didattica. Brescia: La Scuola.

Rivoltella, P.C. (2015). Insegnare al tempo dei "nativi digitali”. Educazione, apprendimento e nuove tecnologie a casa e a scuola dagli asili nido in su. Conferenza condotta dall'associazione cultura La Bottega dell'Arte in occasione della Fiera di Primiero, presso il Teatro di Pieve, Trento.

Rivoltella P. C. (2016). Che cos'è un EAS. L'idea, il metodo, la didattica. Brescia: La Scuola.

Rivoltella, P. C. (2020). Tecnologia più condivisione: così si può fare buon e-learning. Milano: Avvenire.it del 17, Marzo.

Rossi P.G., Pezzimenti L. (2014). La trasposizione didattica. In: Rossi P.G. e Rivoltella P.C., a cura di, L'agire didattico. Brescia: La Scuola.

Rossi P.G. (2011). Didattica enattiva. Complessità, teorie dell'azione, professionalità docente. Milano: FrancoAngeli.

Rossi P.G., Rivoltella P.C., a cura di (2017). L'agire didattico. Brescia: La Scuola.

Seow P., Boticki I., Chia G. (2019). Designing and Implementing Seamless Learning with Teachers. Professionalità Studi, 4, Edizioni Studium, La Scuola: Brescia, pp. 26-50.

Sibilio M. (2011). Corporeità didattiche: i significati del corpo e del movimento nella ricerca didattica. In: Sibilio M., a cura di, Il corpo e il movimento nella ricerca didattica. Indirizzi scientifico-disciplinari e chiavi teorico-argomentative. Napoli: Liguori, pp. 47-69.

Sibilio M. (2012). Corpo e cognizione nella didattica. In: Rivoltella P.C. e Rossi P.G., a cura di, L'agire didattico. Manuale per l'insegnante. Brescia: La Scuola.

Sibilio M. (2012a). La dimensione semplessa delle corporeità didattiche per fronteggiare i bisogni educativi speciali. In: Rivoltella P.C. e Rossi P.G., a cura di, La dimensione pedagogica ed il valore inclusivo del corpo e del movimento. Lecce: Pensa, pp. 9-10.

Sibilio, M. (2012b). La dimensione semplessa dell'agire didattico. Sibilio M, a cura di, Traiettorie non lineari nella ricerca nuovi scenari interdisciplinari. Lecce: Pensa, pp.10-14.

Sibilio M. (2014). La didattica semplessa. Napoli: Liguori.

Sibilio M. (2014a). La dimensione plurare degli studi su corpo e cognizione. In: Rossi P.G. e Rivoltella P.C., a cura di, L'agire didattico. Brescia: La Scuola.

Sibilio M. (2015). Le corporeità didattiche in una prospettiva semplessa. In: Sibilio M. e D'Elia F., a cura di, Didattica in movimento. L'esperienza motoria nella scuola primaria. Brescia: La Scuola Editrice, pp.11-20. 
Sibilio M. (2015b). Simplex didactics: a non-linear trajectory for research in education. Revue de synthèse, 6 (3-4): 477-493.

Sibilio M., Zollo, I. (2016). The non-linear potential of didactic action. Education Sciences \& Society-Open Access Journal, 7(2).

Sibilio M. (2017). Vicarianza e didattica. Corpo, cognizione, insegnamento. Brescia: La Scuola.

Sibilio M. (2020). L'interazione didattica. Brescia: Morcelliana.

Striano M. (2001). La razionalità riflessiva nell'agire educativo. Milano: Garzanti.

Todino M.D., Aiello P., Sibilio M. (2016). Flexible classrooms for inclusive education. ICERI 9th International Conference of Education, Research and Innovation, pp. 1674-1678.

Trentin G. (2019). Apprendimento senza soluzione di continuità negli spazi ibridi dell'infosfera. Professionalità Studi, 4, Edizioni Studium, La Scuola: Brescia, pp.825.

Von Bertalanffy L. (1983). Teoria generale dei sistemi. Fondamenti, sviluppo, applicazioni. Milano: Mondadori. 